\title{
Glass Tube Capillary Arcs in Copper Vapor
}

\author{
Eruzada Maira* Student Member \\ Satoaki Arai* Member
}

The arcing behavior under the circumstance of rich copper vapor in a capillary glass tube were experimentally observed by currents and voltages and spectroscopic method. The arc voltage is affected by the inner diameter of glass tube, the higher arc voltage is, the narrower inner diameter of glass tube is, under otherwise same experimental conditions. It is supposed by the spectral measurement that the arc voltage in a capillary glass tube depents on the arrangement of copper vapor surrounding the arc column. It seems reasonable to consider that the arc voltage increases higher according to the copper vapor layer surrounding arc column forcing the ac current channel to narrow as the inner diameter of glass tube is rendered smaller.

Keywords: capillary arcs, high arc voltage, line spectral distribution, continual spectral distribution

\section{Introduction}

The last purpose of this research is to understand the high arc voltage generation during breaking heavy current of current-limiting fuses ${ }^{(1)}{ }^{(2)}$. This paper describes the experimental observations of characeristics of current and voltage, spectral radiation from electric discharge space and the arrangement of copper vapor layer in the smallest inner diameter of glass tubes estimated by the continuous spectral radiation.

Asample which was constisted of a copper wire element stretched in a glass tubes was put into a pressure container. A heavy current is applied to an apparatus. Arc voltage, current, and spectrum from electric discharge space were measured ${ }^{(3)}$. Two diameters of copper wire were used. In order to investigate the absorption of the light by the thin copper film of the copper adhering to the inner wall of the glass tube after the experiment, the penetration spectrum of white light source was also observed.

The radiation temperature and the particle density distribution of copper vapor layer surrounding arc column were calculated using the continuous spectrum distribution observed for the glass tube of $1 \mathrm{~mm}$ in diameter. The relation between arc voltage and the structure of the arc discharge space in the glass tubes was considered due to the above results.

\section{The Circuit, Apparatus, Spectrum Measurement System and Samples}

2.1 Experimental Circuit An experimental circuit was constructed as shown in Fig. 1 . The current breaking experiment was conducted as follows. The charged capacitor bank $\mathrm{C}$ of $0.136 \mathrm{~F}$ was discharged through the reactor $\mathrm{L}$ of $1.96 \mathrm{mH}$, and breaker $\mathrm{B}$ broke the capacitor bank near the maximum value of the current. The LR discharge circuit which is consisted of the

\footnotetext{
* Tokyo Denki University

2-2, Kanda Nishiki-cho, Chiyoda-ku, Tokyo 101-8457
}

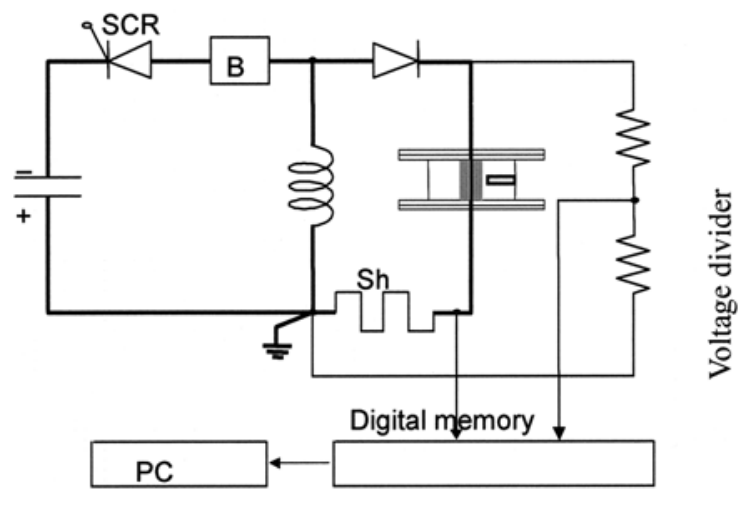

Fig. 1. Experimental circuit

reactor $\mathrm{L}$, the experimental apparatus and the current shunt Sh is constituted, and an arc initiates after a copper wire element melts.

2.2 Experimental Apparatus and Spectral Measurement System The experimental apparatus is consisted of the stainless steel circular disks of $15 \mathrm{~mm}$ in thickness, an insulation cylinder, and an experimental sample. The measurement system of the radiation spectrum from electric discharge space is consisted of the Photonic multichannel analyzer-10 (hereafter call PMA), the optical system controller, and a personal computer. PMA can measure simultaneously the spectrum of wavelength in the range of $300-800 \mathrm{~nm}$ under $\mathrm{t}$ he minimum exposure of $25 \mathrm{~ms}$.

2.3 Experimental Samples The dimensions of Pyrex glass tubes were $50 \mathrm{~mm}$ in length and 1.0, 1.5, $2,2.5,3,3.5 \mathrm{~mm}$ in inner diameter. The material composition of the Pyrex glass tube is $80.1 \mathrm{SiO}_{2}, 12.7 \mathrm{~B}_{2} \mathrm{O}_{3}$, $4 \mathrm{Na}_{2} \mathrm{O}, 2.8 \mathrm{AL}_{2} \mathrm{O}_{3}, 0.04 \mathrm{~K}_{2} \mathrm{O}$, and $0.03 \mathrm{Fe}_{2} \mathrm{O}_{3}$.

The copper wire elements were $0.18 \mathrm{~mm}$, and $0.26 \mathrm{~mm}$ in diameter. The copper purity of the element was $99.9 \%$ 。 The samples were constructed that a copper wire element was stretched in the glass tube with copper electrodes at the both ends. 


\section{Experimental Results}

3.1 Current Breaking Performance and Spectral Radiation for the Elements of $0.18 \mathrm{~mm}$ in Diameter Fig. 2 shows typical current and voltage waveform observed in the experiment. The distributions of spectral intensity according to the wavelength obtained by PMA corresponding to them are shown in Fig. 3. The experiments were conducted using the same melting current throughout.

Fig. 2(a) shows the current and voltage obtained by the glass tube of $1 \mathrm{~mm}$ in diameter. The arc voltage goes up the maximum voltage of $1.9 \mathrm{kV}$ at the arc initiation, after that it decreases with time. The arc voltage waveform has the rectangular shape on average.

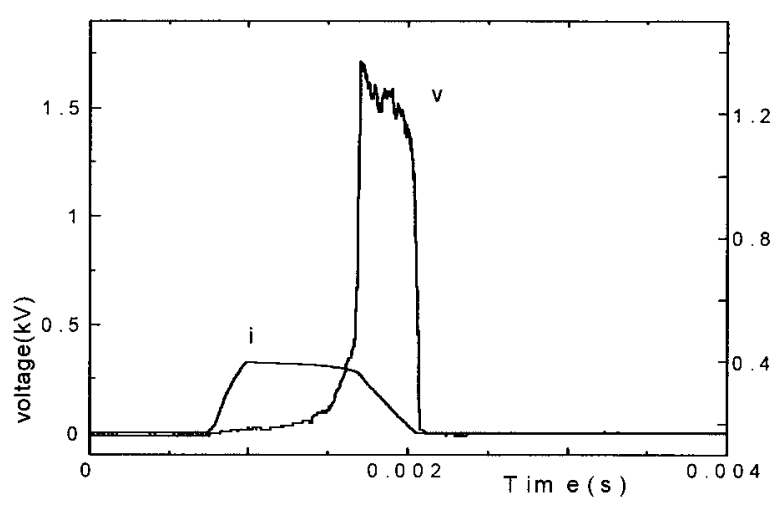

(a) Glass tube of $1 \mathrm{~mm} \phi$

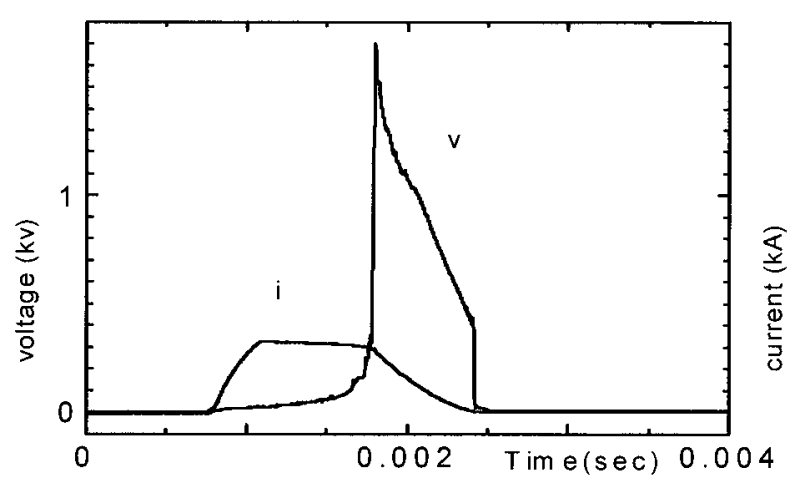

(b) Glass tube of $1.5 \mathrm{~mm} \phi$

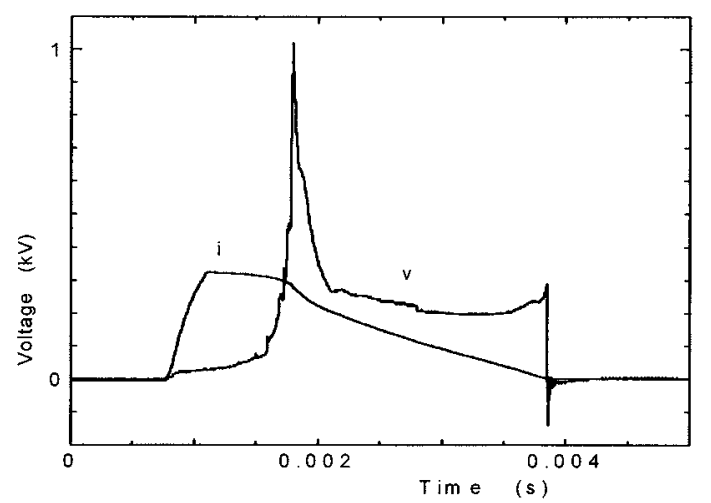

(c) Glass tube of $3.5 \mathrm{~mm} \phi$

Fig. 2. Current and voltage waveform
Fig. 2(b) shows the current and voltage obtained using the glass tube of $1.5 \mathrm{~mm}$ in diameter. As shown in Fig. 2(b), the arc voltage reached $1.7 \mathrm{kV}$ at the arc ignition, and it decreased almost linearly after that until current interception and the voltage waveform looks like an acute angled triangle.

Fig. 2(c) shows the current and voltage obtained using the glass tube of $3.5 \mathrm{~mm}$ in diameter. When the narrower the diameter of the tube is, the higher the arc voltage becomes, therefore the action of current limiting is strong, so the arcing time is shorter. The inner diameter of a tube becomes wider, the arc voltage becomes lower and the arcing time is longer.

The shape of arc voltage is rectangular for the small glass tube diameter, an acute angled triangle for the

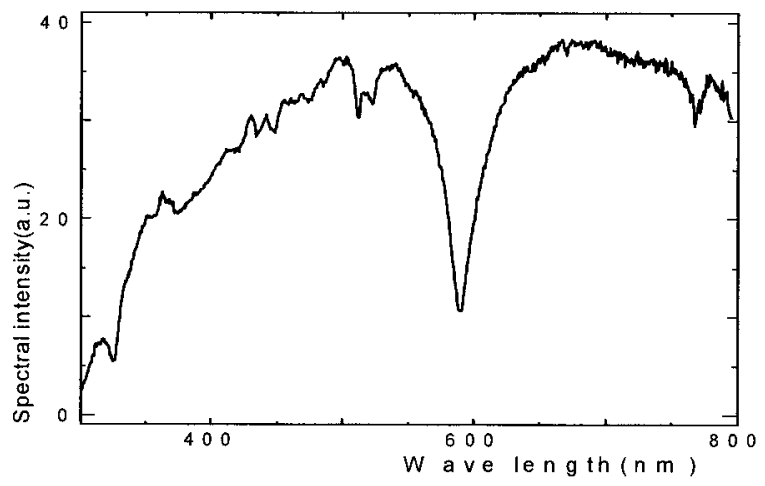

(a) Glass tube of $1 \mathrm{~mm} \phi$ wave length

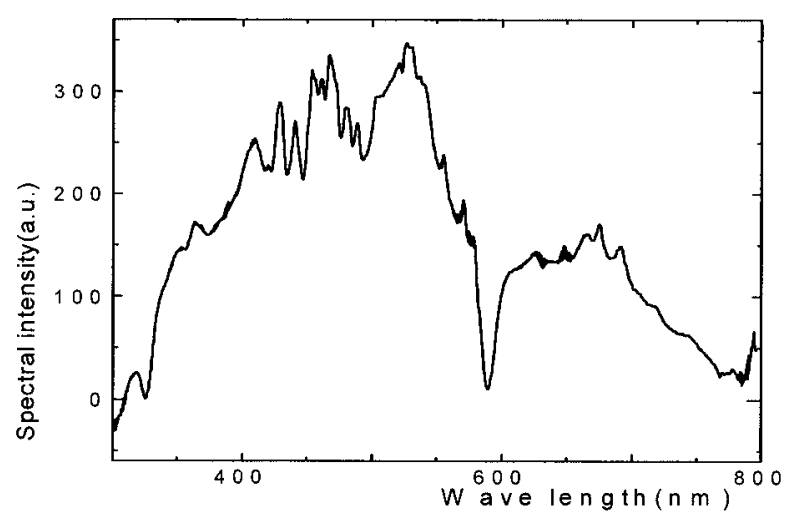

(b) Glass tube of $1.5 \mathrm{~mm} \phi$ wave length

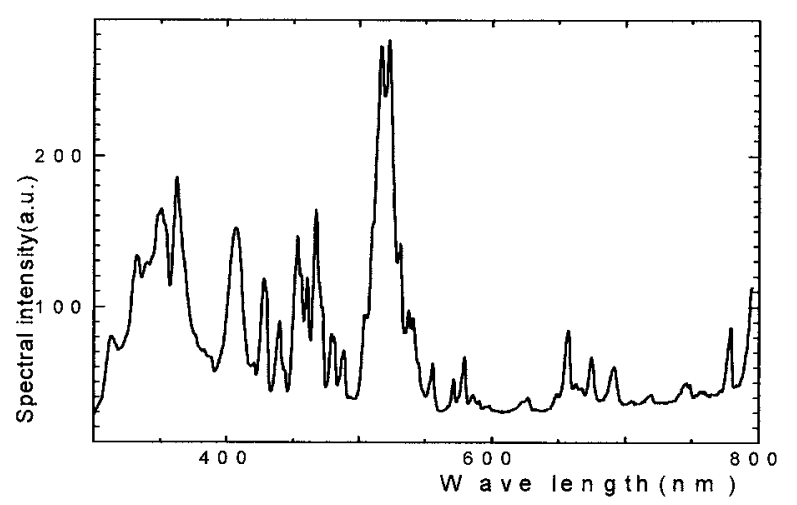

(c) Glass tube of $3.5 \mathrm{~mm} \phi$ wave length

Fig. 3. Copper of $0.18 \mathrm{~mm} \phi$ Spectral wavelength and intensity 
medium diameter, and flat for the larger diameter.

Fig. 3(a) was obtained using the glass tube of $1 \mathrm{~mm}$ in diameter. The measured spectral distribution is mostly continuous and strong absorption is observed about $325 \sim 327 \mathrm{~nm}$ and about $590 \mathrm{~nm}$.

Fig. 3(b) was obtained using the glass tube of $1.5 \mathrm{~mm}$ in diameter. The spectral distribution shows continuous spectral distribution slightly superimposed on the copper spectral line including strong absorption about $325 \sim 327 \mathrm{~nm}$ and about $590 \mathrm{~nm}$.

Fig. 3(c) was obtained using the glass tube of $3.5 \mathrm{~mm}$ in diameter, and copper spectral lines were observed. Spectrum intensity appears significantly strong near $515 \sim 522 \mathrm{~nm}$, and the continuation spectrum decreases remarkably.

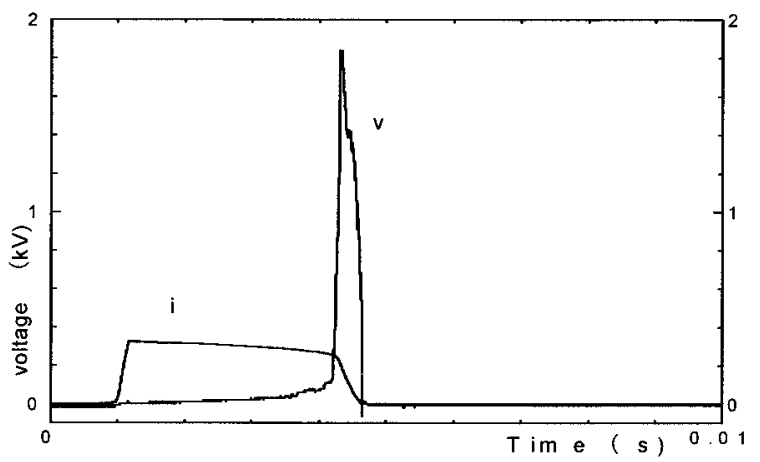

(a) Glass tube of $1 \mathrm{~mm} \phi$

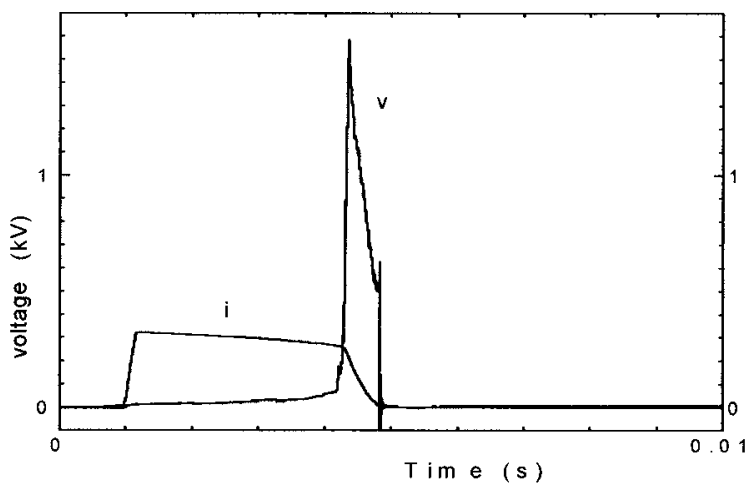

(b) Glass tube of $1.5 \mathrm{~mm} \phi$

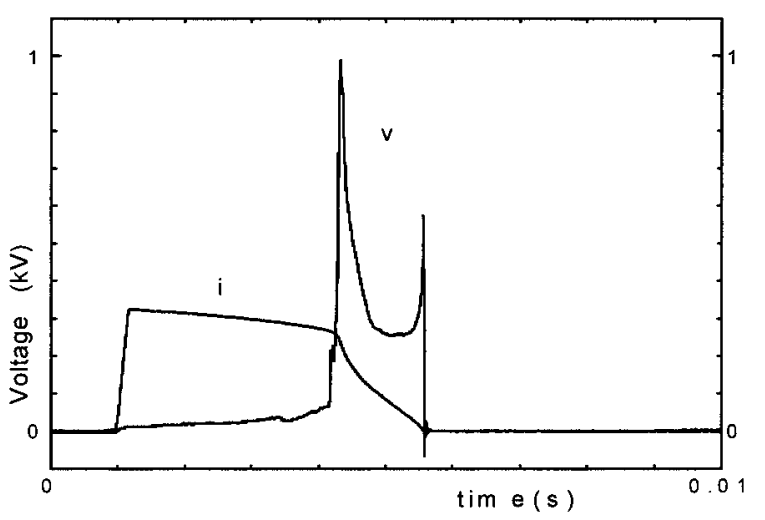

(c) Glass tube of $3.5 \mathrm{~mm} \phi$

Fig. 4. Current and voltage waveform on the copper wire elements of $0.26 \mathrm{~mm} \phi$
3.2 Current Breaking Performance and Spectruam Radiation for the Elements of $0.26 \mathrm{~mm}$ in Diameter Fig. 4 shows typical currents and voltages, which are classified from the feature of the voltage waveform observed in the experiment. The distributions of spectral intensity according to the wavelength obtained by PMA are shown in Fig. 5. The current at melting instant is the same and the energy spent on the tested sample during melting and arcing times is $170 \mathrm{~J} \sim 200 \mathrm{~J}$.

Fig. 4(a) shows the current and voltage obtained using a glass tube of $1 \mathrm{~mm}$ in diameter. The high arc voltage of $1.85 \mathrm{kV}$ occurred immediately after the arc initiation and the arc voltage decreases linearly swelling upwards slightly until the arc current was intercepted.

Fig. 4(b) shows the current and voltage obtained

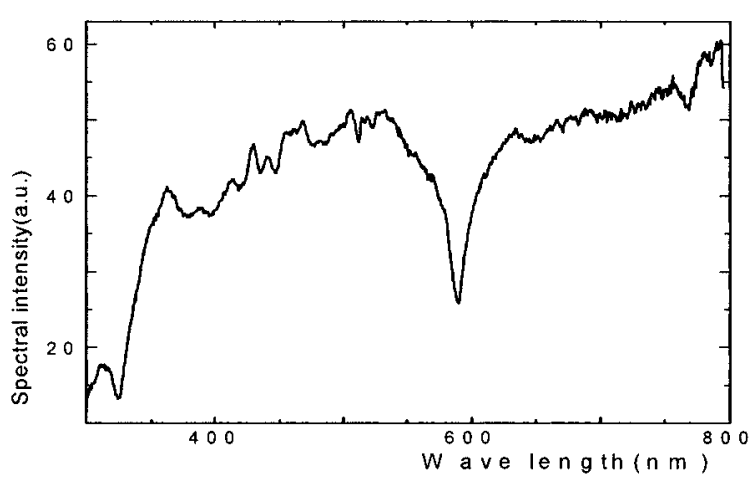

(a) Glass tube of $1 \mathrm{~mm} \phi$

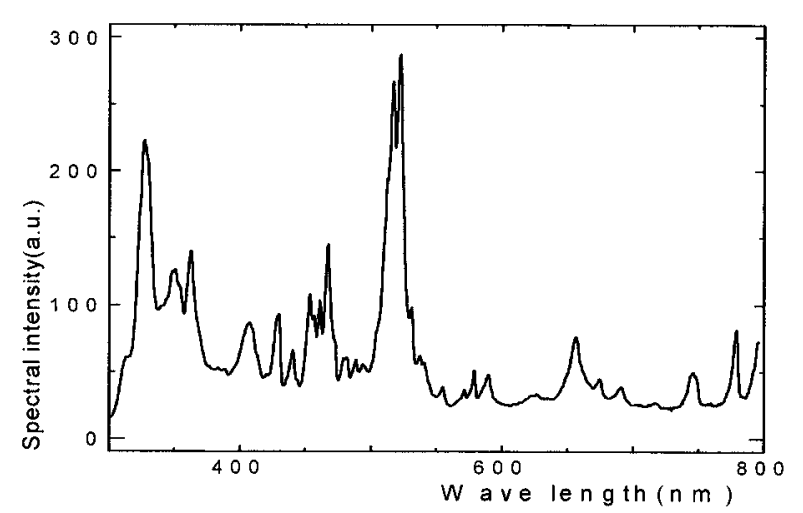

(b) Glass tube of $1.5 \mathrm{~mm} \phi$

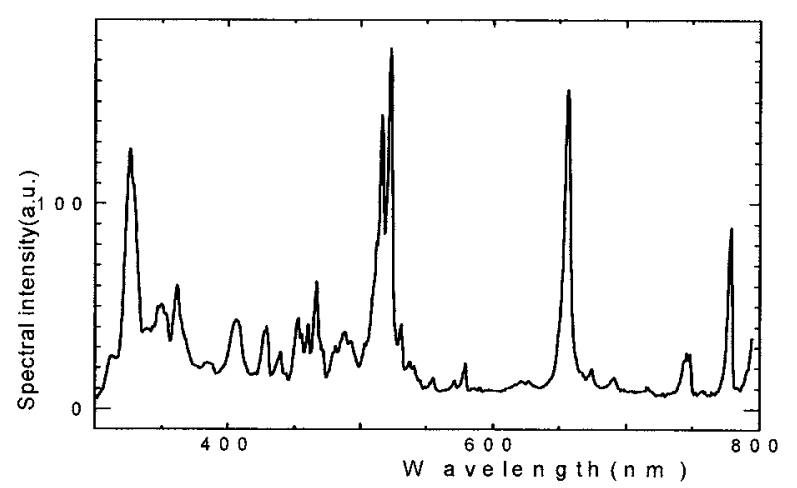

(c) Glass tube of $3.5 \mathrm{~mm} \phi$

Fig. 5. Relation between spectral wavelength and intensity on the copper wire elements of $0.26 \mathrm{~mm} \phi$ 
using a glass tube of $1.5 \mathrm{~mm}$ in diameter, the arc voltage $1.6 \mathrm{kV}$ occurred immediately after the arc initiation and the arc voltage decreased linearly until the arc current was intercepted.

Fig. 4(c) shows the current and voltage obtained using a glass tube of $3.5 \mathrm{~mm}$ in diameter, the arc voltage decreased linearly and then kept constant in a while arc voltage increases about $0.2 \mathrm{msec}$ before current zero, and the peak voltage of $66 \%$ of the arc voltage at the instant of melting appeared at current zero.

Fig. 5 shows the relation between the spectral wavelength and intensity measured simultaneously the currents and voltages of Fig. 4(a) (c).

Fig. 5(a) was obtained using the glass tube of $1 \mathrm{~mm}$ in diameter. Measured spectral distribution is mostly continuous and strong absorption is observed at about $325 \sim 327 \mathrm{~nm}$ and about $590 \mathrm{~nm}$.

Fig. 5(b) was obtained using the glass tube of $1.5 \mathrm{~mm}$ in diameter. Line spectral distribution becomes dominant, and spectral absorptions at $325 \sim 327 \mathrm{~nm}$ and around $590 \mathrm{~nm}$ disappear.

Fig. 5(c) was obtained using the glass tuber of $2.5 \mathrm{~mm}$ in diameter, line spectral was observed.

As experimental results, the arc voltage strongly depends on the cross section of the arc discharge channel, the arc voltage increases corresponding to decreasing the cross section of glass tube.

As recognizing from Fig. 3(b) and Fig. 5(b), spectral radiation changed from the continuous distribution to line according to the decreasing arc energy of each unit length of the copper wire element.

3.3 White Light Penetration of the Pyrex Glass Tube Coated with a Thin Copper Layer

Fig. 6(a) shows the spectral distribution of the light source and the penetrated spectral distribution through the virgin Pyrex glass tube. These spectral distributions are mostly blackbody radiation. The penetrated spectral intensity of the virgin glass tube is less than the spectral intensity of the light source.

Fig. 6(b) shows the penetrated spectral distributions of the Pyrex glass tube of $1 \mathrm{~mm}$ in diameter, and the virgin Pyrex glass tube. The spectral distributions marked (2) were obtained in the current breaking experiment for a copper wire element of $0.18 \mathrm{~mm}$ in diameter, and also the (3) was obtained by the copper wire of $0.26 \mathrm{~mm}$ in diameter.

The penetrated spectral distributions of (2) and (3) are clearly less than that of the virgin Pyrex tube. The spectral intensity distribution of (2) and (3) are same in the range of $300 \sim 550 \mathrm{~nm}$, and the intensity of (3) is slightly larger than one of (2) in the $550 \sim 630 \mathrm{~nm}$, and intensity of (2) is larger than that of (3) over $630 \mathrm{~nm}$. Although resonance absorption of sodium atom appeared near the wavelength of $590 \mathrm{~nm}$ on the spectral distributions obtained during arcing time, it is not observed in the glass tube on which the thin copper layer is coated.

Experimental result of Fig. 6 shows that sodium atoms included in the copper thin film deposited on the wall of glass tube and composed the glass tube wall do not give rise to the clear absorption of light.

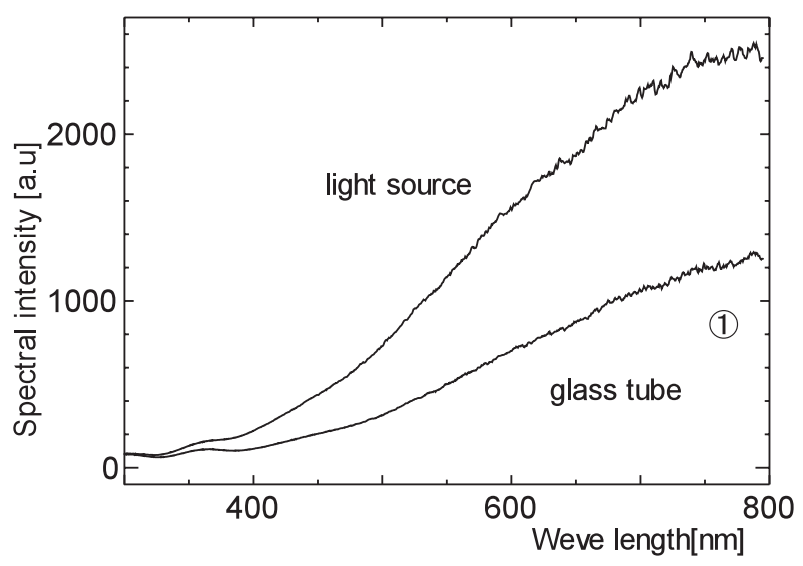

(a) Spectral distribution of light source and penetrated light through the virgin Pyrex glass tube

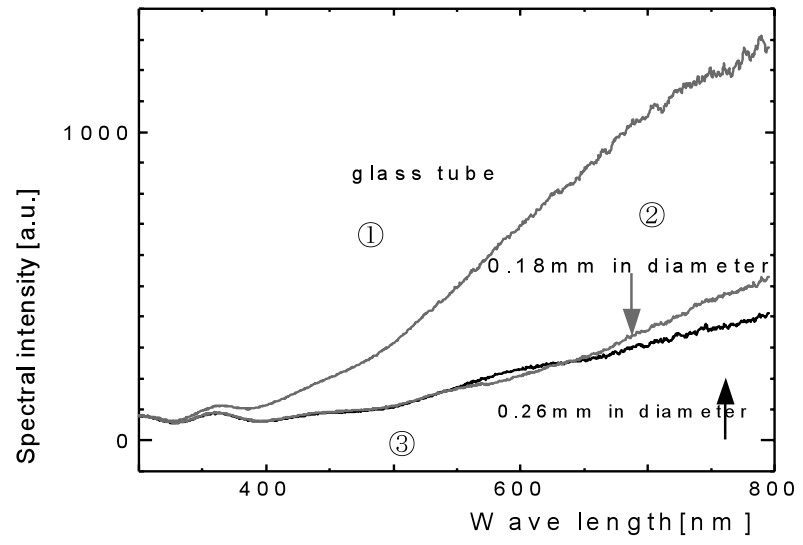

(b) Penetrated spectral distributions through the virgin tubes and the coated with a thin copper film

(1) Penetrated light intensity of virgin glass tube

(2) Penetrated light intensity of glass tube with a thin film by using copper wire element of $0.18 \mathrm{~mm}$ in diameter

(3) Penetrated light intensity of glass tube with a thin film by using copper wire element of $0.26 \mathrm{~mm}$ in diameter

Fig. 6. Penetrated light spectral distributions of white light through the virgin Pyrex glass tube and the same tubes coated with a thin copper film

\section{Structure of Electric Discharge Space}

4.1 Continuous Spectral Distribution The radiation and the absorption for the continual spectral distribution observed on the glass for the continual spectral distribution observed on the glass tube of $1 \mathrm{~mm}$ in diameter are analyzed. Considering the model given by the continuous spectral distribution of black body radiation, and furthermore resonance absorption of about $325 \sim 327 \mathrm{~nm}$ with the thin layer of copper atom and around $590 \mathrm{~nm}$ due to the thin layer of sodium atom, numerical calculation was made about the relation between spectral intensity and wavelength of Fig. 7 Comparing the results of numerical computation of black body radiation with observed continuous spectral distribution gives the temperature of black body and optical lengths and atom densities of sodium and copper.

Radiation intensity of black body is given as follows.

$$
B_{\lambda}=\frac{2 h c^{2}}{\lambda^{5}} \frac{1}{\exp (h c / \kappa \lambda T)-1} \cdots \cdots \cdots \cdots \cdots
$$




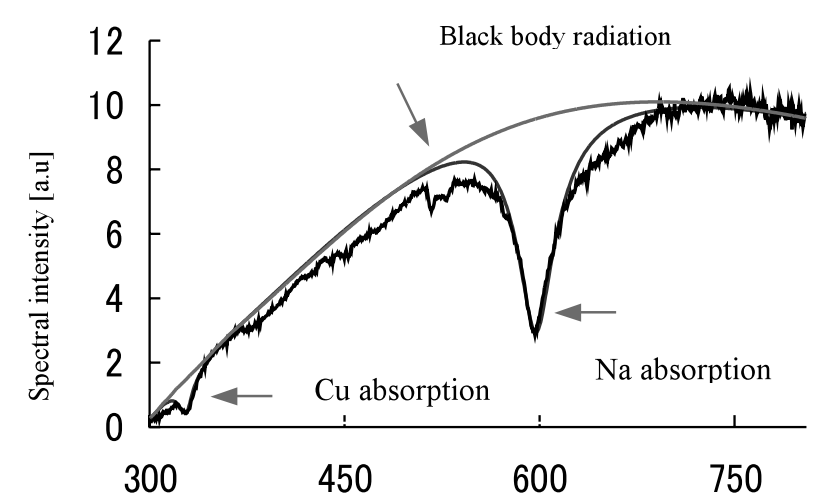

Fig. 7. Spectral distribution and calculated one for the Pyrex glass tube of $1 \mathrm{~mm}$ in diameter

where $\lambda$ : wavelength, $T$ : black body temperature, $h$ : Planck constant, $c$ : velocity of light, and $\kappa$ : Boltzman constant. Moreover, using the law of Lambert radiation expressed as follows

$$
\begin{aligned}
I_{\lambda}= & B_{\lambda} \exp \left[-\left(\alpha_{\lambda N a 1}+\alpha_{\lambda N a 2}\right) l_{N a}\right] \\
& \times \exp \left[-\left(\alpha_{\lambda C u 1}+\alpha_{\lambda C u 2}\right) l_{C u}\right]
\end{aligned}
$$

where $l_{N a}$ : the thickness of a sodium absorption layer, $l_{C u}$ : the thickness of a copper absorption layer, $\alpha_{\lambda N a 1}$ : the resonance line wavelength $589.0 \mathrm{~nm}$ of sodium, $\alpha_{\lambda N a 2}$ : the absorption coefficient of $589.6 \mathrm{~nm}, \alpha_{\lambda C u 1}$ : the resonance line wavelength $324.8 \mathrm{~nm}$ of copper, and $\alpha_{\lambda C u 2}$ : the resonance line wavelength $327.4 \mathrm{~nm}$ of copper.

$\alpha$ is expressed by following equation

$$
\alpha=\frac{1}{4 \pi \varepsilon_{0}} \frac{\pi e^{2}}{m c} N f p_{\lambda}
$$

where $e$ : electron charge, $m$ : electron mass, $N$ : atom density, $\lambda_{0}$ : wavelength $f$ : absorption vibrator strength, $p_{\lambda}$ : profile of spectral intensity distribution

$p_{\lambda}$ is expressed by the Lorentz type function and is given as follows

$$
p_{\lambda}=\frac{1}{\pi} \frac{\gamma / 2}{\left(c / \lambda-c / \lambda_{0}\right)^{2}+(\gamma / 2)^{2}}
$$

where $\gamma$ is the value of half width of the resonance line given by Margenau and Watson ${ }^{(3)}$, and is given as follows

$$
\gamma=\frac{e^{2} f N \lambda_{0}}{8 \pi^{2} m c} \ldots \ldots \ldots \ldots \ldots \ldots \ldots \ldots \ldots \ldots \ldots \ldots
$$

Calculation using equations (1) to (5) gives the continuous spectral distribution including resonance absorption by copper and sodium atoms. The profile of a continual spectral distribution depends on the temperature.

The vibrator intensity used for calculation is $f_{N a 1}=$ $0.655, f_{N a 2}=0.327, f_{C u 1}=0.320, f_{C u 2}=0.160^{(4)}$.

The calculation results give the black body temperature of $4300 \mathrm{~K}$, the atom density of copper layer $N_{C u}=2.3 \times 10^{27}\left[\mathrm{~m}^{-3}\right]$ copper layer optical length $l_{C u}=1.8 \times 10^{-8}[\mathrm{~m}]$ and atomic density of sodium

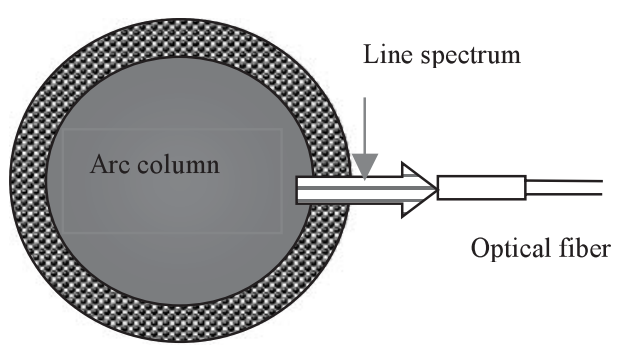

(a) Large diameter of a glass tube

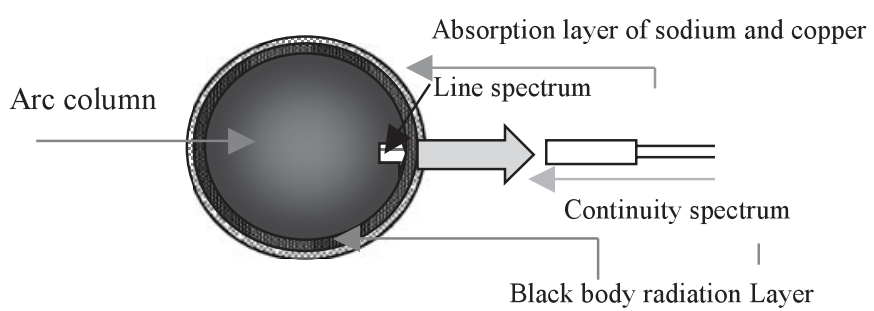

(b) Small diameter of a glass tube

Fig. 8. Particle distribution depending on the diameter of glass tube

layer $N_{N a}=6 \times 10^{26}\left[\mathrm{~m}^{-3}\right]$, sodium optical length $l_{N a}=2.8 \times 10^{-8}[\mathrm{~m}]$. The distribution model of copper vapor and cluster in the discharge space is given as shown in Fig. 8(b) from the above analytical results. For the small diameter glass tube, the continuous spectral distribution accompanied by the absorption of copper and sodium was observed, but the spectrum from an arc column was not able tocannot be observe directly.

The layer of copper and sodium vapor with characteristics of the black body radiation and absorption surrounds the arc column.

The constitution of the black body radiation layer of glass tube of $1 \mathrm{~mm}$ in diameter is considered that the parts of copper atoms around an arc column condense in clusters and the optical dense layer is formed by the copper vapor and clusters and the layer radiates the black body radiation. When the diameter of glass tube is wide, it is thought that the density of copper vapor in a glass tube is low, and the layer consisting of copper vapor and around an arc column is of low density, the line spectrum from an arc column of a central tube domain penetrates through the copper vapor layer and the tube wall.

4.2 Effect of the Arc Energy As recognizing from Fig. 3(b) and Fig. 5(b) obtained by the experimental results used the glass tube of $1.5 \mathrm{~mm}$ in inner diameter, elements of 0.18 and $0.26 \mathrm{~mm}$ in diameter and the same charged voltage of source capacitor bank of experimental circuit, spectral distribution radiation changed from continuous distribution for $0.18 \mathrm{~mm}$ diameter element to line for $0.26 \mathrm{~mm}$ diameter element.

Arc initiation current was $300 \mathrm{~A}$ for Fig. 2(b) and $266 \mathrm{~A}$ for Fig. 4(b), arc energy consumed by each experiment was $88 \mathrm{~J}$ for Fig. 2(b) and $69 \mathrm{~J}$ for Fig. 4(b).

Arc energy of each unit length of the copper wire element for Fig. 4(b) is $20 \%$ of Fig. 2(b) because the cross section of element for Fig. 4 (b) is 4 times that of Fig. 2(b).

From the experiment results, it is estimated that the evaporated copper element mass for Fig. 4(b) is less than 
that for Fig. 2(b) depending on the arc energy. It is supposed that the remaining mass of liquefied element breaks down many droplets, they are between the arc column and the wall of glass tube or on the wall of glass tube, so that, optical state of outside space of the arc column is optically thin, spectral emissions of arc column mostly come through directly the glass tube.

\section{Conclusions}

The arc voltage strongly depends on the cross section of the arc discharge channel, the arc voltage increases corresponding to decreasing the cross section of glass tube.

The continuous spectral distribution was observed for the radiation of arc column in Pyrex glass tube of $1 \mathrm{~mm}$ in inner diameter and copper wire element of $0.18 \mathrm{~mm}$ and $0.26 \mathrm{~mm}$ in diameter. Furthermore, remarkable absorption was observed around sodium radiation wavelength of $590 \mathrm{~nm}$ and copper radiation wavelength of $325 \sim 327 \mathrm{~nm}$.

For the glass tube diameter of $1.5 \mathrm{~mm}$ and the copper wire element diameter of $0.18 \mathrm{~mm}$ and $0.26 \mathrm{~mm}$ the continuous spectral intensity decrease and the line spectral intensity increases remarkably with glass tube diameter becoming larger. On one hand, high arc voltage is generated at the initiation of arc, and decreases linearly with time. The peak arc voltage appeared just before arc current zero for the glass tubes of $3.5 \mathrm{~mm}$ in diameter.

Sodium atoms included in the copper thin film deposited on the wall of glass tube and composed the glass tube walls do not give rise to the clear absorption of light.

The radiation during arcing time of glass tube of $1 \mathrm{~mm}$ in diameter and copper wire element of $0.18 \mathrm{~mm}$ in diameter was simulated in consideration of spectral absorption of copper and sodium. It is considered that an arc column is surrounded by the layer of optically dense copper vapor clusters and droplets, the radiation from the arc column is absorbed by the layer, then the black body radiations are performed by the layer, which absorbs furthermore the absorption layer of copper and sodium is outside the black body radiation layer.

The constitution of discharge media distribution around arc column is proposed for glass tube of $1 \mathrm{~mm}$ in diameter and copper wire element of $0.18 \mathrm{~mm}$ in diameter.

It is estimated that the evaporated mass of large diameter element is less than that of smaller one under the conidian of the same charged voltage of source capacitor bank of experimental circuit.

It is supposed that the remaining mass of liquefied element breaks down many droplets, they are between the arc column and the wall of glass tube or on the wall of glass tube, so that, optical state of outside space of the arc column is optically thin, spectral emissions of arc column mostly come through directly the glass tube.

(Manuscript received Feb. 28, 2003,

revised Oct. 2, 2003)

\section{References}

(1) S. Arai and S. Hamada: "Experimental investigation of capillary arc phenomena", Proc. ICEFA, pp.236-242, Ilumenau, Germany (1995)

(2) S. Arai and S. Hamada: "Temperature Estimation of arc in Glass Capillary by spectroscopic Measurement", Proc. SAP \& ETEP-97, pp.253-257, Lodz, Poland (1997)

( 3 ) H. Margenau and W. Watson: "Pressure Effects on Spectral Lines", Rev. Mod., Vol.8 (1936)

(4) H. Daidouji and T. Nakahara: Atomic Spectra Measurment and their Applications (1989) (in Japanese)

(5) B. Weber and R. Scholl: "A Noble Type of Light Source Continuosu Radiation from Small Clusters in Microwave Excited Discharges", J. IES, Summer, 93 (1992)

Eruzada Maira (Student Member) was born on July 6,

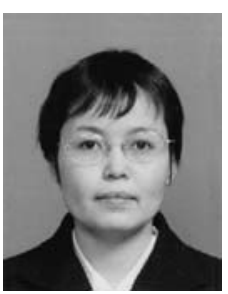
1970. Received a M.E. degree in Electrical Engineering from Tokyo Denki University in 2003. She is Presently working towards the Ph.D. degree at the same university. Her current research field is the arcing Phenomea in the high voltage switching devices.

Satoaki Arai (Member) received the Ph.D. degree in electrical engineering from Tokyo Denki University in 1968. Presently, he is a Professor at Tokyo Denki University. He is currently interested in the current limiting fuses, high voltage switches for power distribution system. 